FEATURE

\title{
Looking to the
}

\section{future of oral}

\section{health care}

A preview of an important topic that will be

discussed this November at the British Society of

Dental Hygiene and Therapy's (BSDHT's) Oral Health

Conference 2019 in Wales. 
$\mathrm{I}$

n 2015, Wales introduced the Well-being of Future Generations Act. A ground-breaking piece of government legislature, this Act has revolutionised the way that industry and society operate in the country, and is having a profound impact on everything from healthcare to the arts.

As part of the exciting educational programme available at the Oral Health Conference 2019 (OHC), the British Society of Dental Hygiene and Therapy (BSDHT) has invited Andrew Rogers, a lecturer at the University of Manchester who lives and works from his home in North Wales, to give a session focused on this interesting piece of legislature. In this talk he will examine how it could help influence and support a number of important improvements in oral health.

Entitled 'Oral Health Future Generations in Wales - What Does the Well-being Act Offer?' this informative lecture is the perfect opportunity for delegates to think outside the box and consider how this policy could work in their favour.

\section{Understanding the Act}

So, what are the core aims of the Act?

Andrew Rogers says: 'The main point of this legislation is to encourage people on a national scale to think about how their actions will impact future generations, instead of just considering the short-term effects. The Act has introduced seven well-being goals, and each of these have been created in order to inspire different sectors to change their way of thinking, their actions and the influence they have.

'Some of these goals, such as the quest for A Healthier Wales or A More Equal Wales, understandably seem to be much within the remit of certain industries - eg a Healthier Wales will primarily concern healthcare providers. However, what I want to help people realise is that the role of different sectors will overlap when it comes to achieving all these goals, and this will open up many opportunities to finally get important messages heard. For example, although healthcare industries will be focusing on people's well-being, there's no reason why they shouldn't be lessening their impact on the environment and embracing arts and culture as well.

'This is just a very simple example, but if dental practices become more forwardthinking about their waste and proper disposal, and start to consider how things such as single use plastics and dental amalgam are impacting the environment, it's a good first step to take towards a more sustainable way of operating. This will help towards achieving the goal of keeping the environment safe for future generations.'

He also adds that this is only the first step because much more can be achieved by really embracing the Act and being adventurous with a broader social responsibility. moment, there's the very real possibility that the rest of the UK could later implement it on a wider scale. The Act is being watched closely by nations around the globe and there is mounting pressure on the UK Government to also follow suit. This is a fantastic opportunity for dental hygienists and dental

\section{'This informative lecture is the perfect opportunity}

for delegates to think outside the box and consider how this policy could work in their favour.'

\section{Five aspects of success}

As part of the Well-being Act, the Welsh Government has recommended that these seven goals are supported by five fundamental ways of working that ensure everyone can get involved and reach these goals together. These are as follows: Long-term, Integration, Involvement, Collaboration and Prevention.

'As you can see, these ways of working closely echo the goals that healthcare and dental professionals have already, Andrew comments. 'For example, let's focus on prevention. In dentistry the shift towards preventative care instead of fixing problems caused by bad lifestyle choices or a sugar dominated food system is already in effect, but seeing this confirmed in government legislation is something new. For the first time we are witnessing a government change their way of thinking towards stopping problems before they occur, instead of thinking of ways to pick up the pieces, and thinking of the impact of their actions not tomorrow, this year or maybe next year but in the next generation. This is incredibly exciting!

'This could easily lead to the opportunity for oral health professionals to make a real difference going forward. What about the war against sugar? Water fluoridation? We know that sugar is responsible for a wide array of health conditions and highly detrimental to oral health, and that water fluoridation schemes are successful at lessening tooth decay in areas they are in effect. With legislation like this in action, it's easier for oral health professionals to take greater action on these points and gain support from other bodies, as for once the government is firmly on their side.'

\section{In Wales and beyond}

'Although this Act is only in Wales at the therapists to really consider what changes they want to make and to come up with ways to implement them.

'We all need to work together to create a wider feeling of well-being, and this means that all industries as well as public bodies will have to concentrate on every area in order for this Act to make the greatest amount of impact. I want to inspire people attending my talk to take action, pick up the mantle and realise that the future really is in our hands. We need to work together now to ensure that the world is somewhere people can co-exist not only in the next 30 years, but hundreds of years into the future.'

\section{More at the OHC 2019}

Andrew Rogers'
session is just one
of the exciting
educational
opportunities
awaiting individuals
attending the $\mathrm{OHC}$
this year on $22-23$
November.

You can find out more about the $\mathrm{OHC}$ and register your place by visiting the BSDHT website: http://www.bsdht.org. uk/OHC2019.

For more information about the BSDHT, visit www.bsdht.org.uk, call 01788575050 or email enquiries@ bsdht.org.uk. 